\title{
THE NORMALITY OF A PRODUCT WITH A COMPACT FACTOR
}

\author{
BY MARY ELLEN RUDIN ${ }^{1}$
}

Communicated January, 1973

Assume all spaces are $T_{1}$ and all maps continuous.

Suppose $X$ is compact and $Y$ normal. Even if $X$ is the closed unit interval there is no reason to expect $X \times Y$ to be normal [1]. Classically we have: $X \times Y$ is normal if $Y$ is paracompact [2]; $\beta Y \times Y$ is normal only if $Y$ is paracompact [3]; and $[0,1] \times Y$ is normal if and only if $Y$ is countably paracompact [4]. The purpose of this paper is to announce that the following conjecture of Morita [5], [6] is true.

THEOREM. If $X$ is compact, $X \times Z$ normal, and $Y$ the image of $Z$ under a closed map, then $X \times Y$ is normal.

Partial results have been obtained by $\mathrm{T}$. and $\mathrm{K}$. Chiba.

To show the spirit of this proof we now make some definitions and then give the principal lemmas of the proof.

If $\kappa$ is a cardinal, we say the space $Z$ is $\kappa$-collectionwise normal provided, for all discrete families $\left\{F_{\alpha}\right\}_{\alpha<\kappa}$ of subsets of $Z$, there is a family $\left\{U_{\alpha}\right\}_{\alpha<\kappa}$ of disjoint open sets with $F_{\alpha} \subset U_{\alpha}$ for each $\alpha<\kappa$. We say a family $\left\{G_{\alpha}\right\}_{\alpha<\kappa}$ of subsets of a space $Y$ is hereditarily closure preserving provided each family $\left\{J_{\alpha}\right\}_{\alpha<\kappa}$, with $J_{\alpha} \subset G_{\alpha}$ for each $\alpha$, is closure preserving in $Y$. Suppose $X$ and $Z$ are spaces and $g:(X \times Z) \rightarrow[-1,1]$ is continuous. For $z \in Z$ define $U_{z, g}=\{u \in Z \mid$ for all $x \in X, g(x, u)=-1$ implies $g(x, z)<0$ and $g(x, u)=1$ implies $g(x, z)>0\}$. Then we call $\left\{U_{z, g} \mid z \in Z\right\}$ a separating set for $Z$ with respect to $X$.

Lemma 1 in a way attaches a cardinal function to the fact that $X \times Z$ is normal for a compact $X$ :

LeMma 1. Suppose $X$ is compact, $X \times Z$ is normal, and $\mathscr{U}$ is a separating set for $Z$ with respect to $X$. Then there is a cardinal $\kappa$ such that $Z$ is $\kappa$ collectionwise normal and $\mathscr{U}$ has a locally finite closed refinement of cardinality $\kappa$.

Observe that closed maps preserve $\kappa$-collectionwise normality and take locally finite families into hereditarily closure preserving families. Thus

AMS (MOS) subject classifications (1970). Primary 54B10, 54C10, 54A25; Secondary 54D20, 54D15.

Key words and phrases. Product, normality, compact, closed map.

${ }^{1}$ Partially supported by NSF GP-29537X. 
it is easy to prove that Lemma 2 completes the proof of Morita's conjecture. However I feel Lemma 2, which does not mention products, may be useful in many situations.

LEMMA 2. If $Y$ is $\kappa$-collectionwise normal and an open cover of $Y$ has a cardinality $\kappa$ hereditarily closure preserving closed refinement, then it has a locally finite closed refinement.

\section{REFERENCES}

1. M. E. Rudin, A normal space $X$ such that $X \times I$ is not normal, Fund. Math. 63 (1971), 179-186.

2. J. Dieudonné, Une généralisation des espaces compacts, J. Math. Pures Appl. (9) 23 (1944), 65-76. MR 7, 134.

3. H. Tamano, On paracompactness, Pacific J. Math. 10 (1960), 1043-1047. MR 23 \# A2186.

4. C. H. Dowker, On countably paracompact spaces, Canad. J. Math. 3 (1951), 219-224. MR 13, 264.

5. K. Morita, Paracompactness and product spaces, Fund. Math. 50 (1961/62), 223-236. MR 24 \# A2365.

6. K. Nagami, Normality of products, Proc. Internat. Congress Math. (Nice, 1970), vol. 2, Gauthier-Villars, Paris, 1971, problem 1, p. 33.

7. T. and K. Chiba (to appear).

Department of Mathematics, University of Wisconsin, Madison, Wisconsin 53706 